Service social

\title{
Les politiques sociales et l'équité. Un programme de recherche
}

\section{Pierre-Gerlier Forest}

Volume 46, numéro 1, 1997

Politique et intervention

URI : https://id.erudit.org/iderudit/706747ar

DOI : https://doi.org/10.7202/706747ar

Aller au sommaire du numéro

Éditeur(s)

École de service social de l'Université Laval

ISSN

1708-1734 (numérique)

Découvrir la revue

Citer cet article

Forest, P.-G. (1997). Les politiques sociales et l'équité. Un programme de recherche. Service social, 46(1), 7-28. https://doi.org/10.7202/706747ar

\section{Résumé de l'article}

Cet article expose les conditions nécessaires à l'élaboration d'un programme de recherche comparée sur les politiques sociales, programme dans lequel diverses conceptions de la justice et du bien-être seront examinées et évaluées. L'analyse politique normative permet en fait de juger les objectifs ou les résultats des interventions publiques à la lumière des orientations et des choix qui s'expriment dans les politiques et dans les affrontements à leur sujet. Le problème que pose la réalisation de l'équité dans la vie sociale sert d'illustration pour cette approche, dans une perspective théorique et méthodologique aussi bien que pratique. 


\section{ARTICLES}

\section{Les politiques sociales et l'équité Un programme de recherche*}

Pierre-Gerlier FOREST

Cet article expose les conditions nécessaires à l'élaboration d'un programme de recherche comparée sur les politiques sociales, programme dans lequel diverses conceptions de la justice et du bien-être seront examinées et évaluées. L'analyse politique normative permet en fait de juger les objectifs ou les résultats des interventions publiques à la lumière des orientations et des choix qui s'expriment dans les politiques et dans les affrontements à leur sujet. Le problème que pose la réalisation de l'équité dans la vie sociale sert d'illustration pour cette approche, dans une perspective théorique et méthodologique aussi bien que pratique.

Conditions for the development of a new program of research in the field of social policy are stipulated in this article with the purpose of facilitating the evaluation and comparison of different conceptions of justice and welfare. Normative policy analysis makes practicable a judgment over the objectives and consequences of public policies, as other approaches in policy analysis, but with an emphasis on the particular social choices and orientations that are expressed in public policies, or in political conflicts about policy issues. The question of social equity is used as an illustration for the research approach, on a theoretical and methodological level, as well as in a more practical manner.

* Ce texte a été produit dans le cadre d'une entente entre l'École de travail social de l'Université Conception au Chili et l'École de service social de l'Université Laval. II devait servir de toile de fond à un éventuel programme de recherche sur les liens entre politiques sociales et intervention. Lise Tessier, directrice de l'École, et l'auteur sont allés au Chili en janvier 1996 pour amorcer ce travail. Élaine Carey-Bélanger et Laval Doucet, professeurs ici, sont présentement engagés dans l'enseignement à la maîtrise au Chili et dans la poursuite de l'élaboration du programme de recherche. 
Le texte trouve son origine dans un séminaire organisé à l'initiative du programme de maîtrise en travail social et politiques sociales de l'Université de Concepcion (Chili), entre le 8 et le 12 janvier 1996. Merci à Patricia Aguilera Fiero, Julia Chavez Carapia, Élaine Carey-Bélanger, Manuel Sanchez Rosado, Pedro Vera Castillo et Patricia Witham Kiley, pour leur participation amicale et critique à différentes étapes de cette réflexion.

La question des valeurs embarrasse la recherche sur les politiques sociales, de la même façon qu'elle embarrasse les individus et les groupes engagés dans l'action ou la réforme. Comme l'écrivait Fernand Dumont dans l'introduction du Traité des problèmes sociaux (1995, p. 21), «il n'y a pas de normes toutes faites qui puissent juger des problèmes sociaux et encadrer de façon indubitable l'incessant travail de production du social ». Comment exercer un jugement sur les préférences ou les pratiques sociales sans se heurter à la diversité des codes culturels et moraux ou des prescriptions qui en découlent? Pourquoi remédier aux effets de l'injustice ou de l'exclusion, si les conséquences de l'intervention - fausses attentes, stigmatisation, dépendance, etc. - se révèlent aussi dommageables à long terme que la situation de départ?

En fait, dès lors que les chercheurs s'intéressent sérieusement aux enjeux et aux choix collectifs en matière sociale, toute la rigueur et toute l'objectivité dont aime à se parer la science ne peuvent les préserver de l'indignation et d'un certain sentiment de responsabilité devant les situations les plus révoltantes ou les plus pathétiques : souffrance, isolement, violence, contrainte, dénuement, etc. Même les économistes, qui affichent normalement leur méfiance à l'endroit des jugements de valeur, admettent volontiers qu'ils ne peuvent étudier les problèmes sociaux sans tenir compte des obligations morales des personnes et des institutions, au-delà des considérations habituelles sur l'efficacité et l'efficience (Le Grand et Robinson, 1984). Sauf par quelque illusion ou artifice rhétorique, il est impossible de dissocier l'éthique et l'action sociale; il faut en tirer les conséquences avant d'imaginer toute stratégie de recherche.

Certes, il n'est pas rare de rencontrer des programmes et des projets de recherche dans lesquels les opinions préconçues et les choix idéologiques tiennent lieu de justifications ou de critères d'évaluation. Dans sa typologie des utilisations de la 
recherche sociale, Carol $\mathrm{H}$. Weiss (1986) avait mis au jour au moins quatre situations courantes dans lesquelles les finalités morales et politiques de la recherche passent avant les principes méthodologiques d'objectivité et d'impartialité : (1) l'aide à la décision et à la résolution de problèmes; (2) la contribution aux débats publics; (3) la recherche sous mandat destinée à établir le bien-fondé d'une position politique préalable; (4) l'usage partisan ou "tactique » des résultats scientifiques. Toutes ces situations ne sont pas condamnables, loin s'en faut, et toutes ne conduisent pas à la dégradation des méthodes de travail ou des règles de conduite propres aux chercheurs (Jenkins-Smith et Sabatier, 1992, p. 50-55). Mais il est clair qu'en recherche sociale les préoccupations pratiques prennent aisément le pas sur la production de connaissances fondamentales et, plus largement, sur le postulat de neutralité de la science. On ne peut échapper aux pressions qui s'exercent pour orienter les activités de recherche vers les besoins des acteurs sociaux et politiques, notamment l'État et la communauté, sous peine de marginalisation ou même d'insignifiance (Shensul et Stern, 1985).

L'existence d'une demande sociale forte pour des résultats de recherche concrets et applicables n'est tout de même pas un prétexte pour s'abandonner au plus offrant. II devrait être assez évident que toutes les pressions qui s'exercent ne sont pas nécessaires à l'intelligence des problèmes sociaux et à leur résolution. À la différence de l'arène politique, où il est sans doute important de prévoir des règles pour assurer l'égalité «formelle » des intérêts et des points de vue, l'arène scientifique est régie par des règles et des procédures qui prévoient le déclassement et même le rejet des perspectives erronées ou inutiles. La question de base est donc de savoir comment comprendre et, surtout, comment maîtriser la relation entre politique et recherche, entre pratique et réflexion, entre engagement moral et critique sociale, entre connaissance et intervention, de sorte que les perspectives justes et utiles inspirées par l'expérience contribuent à l'activité intellectuelle et à la quête de solutions désirables (Jenkins-Smith, 1990; Hogwood et Gunn, 1984; Majchrzak, 1984; Aaron, 1978).

Comme on le verra dans ce texte, plusieurs spécialistes de l'analyse des politiques ont soulevé des questions de ce genre, au point de constituer un véritable courant de pensée, à la rencontre de trois traditions différentes : la méthode axiomatique, marquée au coin de la philosophie analytique; l'analyse culturelle, 
d'inspiration sociologique et ethnologique; l'analyse stratégique, en prolongement des travaux sur les systèmes politiques (Forest, 1997a). Toutes ces approches ont en commun de vouloir situer les structures et les institutions qui contribuent à entretenir ou à créer les problèmes sociaux dans le cadre plus vaste d'une réflexion sur les valeurs et les préférences. En conséquence, toutes ces approches proposent des critères pour faire le partage entre les fondements nécessaires de l'action sociale - la structure de base de la société, par exemple, ou les objectifs premiers de l'intervention gouvernementale - et les principes qui relèvent plutôt de la subjectivité ou de la réalité particulière à un groupe ou à une classe.

Pour anticiper la discussion ultérieure, disons que la recherche sur les politiques sociales gagne à s'ouvrir à ces différents critères, en rupture avec la tradition fonctionnaliste encore dominante. À l'instar d'Iris Marion Young (1990), il nous semble en effet qu'en insistant exclusivement sur la distribution des richesses ou des droits, le fonctionnalisme nous a conduits à négliger les enjeux qui sont liés à l'exercice du pouvoir social et aux institutions où il s'incarne, alors que ces deux caractéristiques fondent la structure générale de l'autorité politique et, partant, les obligations et les limitations qui s'appliquent à l'action collective.

Le texte qui suit tente de poser les conditions nécessaires à l'élaboration d'un programme de recherche sur les politiques sociales dans lequel diverses conceptions de la justice et du bien-être peuvent être examinées et évaluées. Cet effort d'analyse politique normative est d'autant plus important que nous vivons dans des sociétés qui sont fragmentées, socialement et culturellement, tant et si bien que l'idée de valeurs communes est contestée. Pourquoi faudrait-il juger les objectifs ou les résultats d'une intervention publique à la lumière de préférences qui ne se trouvent peut-être que dans la conscience et le désir des chercheurs, plutôt qu'à la lumière des orientations et des choix qui s'expriment vraiment dans les politiques et dans les affrontements à leur sujet?

\section{COMPARER POUR COMPRENDRE ET POUR AGIR}

Précisons tout de suite que le pluralisme n'est jamais absolu, quelle que soit la société et bien que la tendance dans cette direction augmente avec la mondialisation et le développement des 
échanges. Pour les groupes opprimés ou dominés, il est même possible que le relativisme culturel et moral qui a cours aujourd'hui apparaisse comme un autre moyen pour faire taire les revendications pour la justice ou l'égalité, en enfermant chacun dans sa différence (Burgi-Golub, 1996; Minow, 1987; WasserStrom, 1980). Si l'on accepte la proposition de Lemieux (1989 et 1995), qui définit le pouvoir comme la capacité d'un acteur de contrôler les décisions qui concernent ses ressources ou celles des autres acteurs, on doit aussi admettre que les règles qui se présentent sous le manteau des valeurs peuvent renforcer les effets abusifs de la stratification sociale ou économique, en justifiant le contrôle des plus puissants sur les plus démunis ${ }^{1}$. La question des valeurs n'est donc pas embarrassante seulement parce qu'elle nous amène à considérer la diversité des codes culturels et moraux, mais encore parce qu'elle nous force à nous interroger sur la nature de la communauté politique, plus ou moins hiérarchique, plus ou moins coercitive, et sur les moyens qui servent à l'intégration et à la contrainte.

Ce n'est pas tout. L'aptitude d'une personne à rendre compte des codes culturels et moraux propres à la société à laquelle elle appartient est toujours limitée, pour des raisons qui sont bien connues des anthropologues:

Staying within his own culture, a person is apt to see no culturally standardized forms around him : transgression against the norm is more visible than conformity. [...] The innocent view of culture is that we don't have it at home; it is only abroad that people are culturally hidebound (Douglas, 1990, p. 4).

Il est donc risqué de s'avancer sur le terrain des valeurs et des politiques sans transformer d'abord le système de référence sur lequel s'appuie l'analyse, sans trouver d'abord un moyen de poser sur notre culture et notre société d'origine un « regard éloigné $^{2}$ » (Lévi-Strauss, 1983). Comment faire autrement? Dans l'analyse des politiques sociales, poser la question des valeurs

1. Dans certaines conditions d'exclusion, du reste, s'opposer à cette tendance et réclamer la conservation du lien social au niveau de la société nationale tout entière est parfois l'unique chance qui demeure de survivre et de vivre.

2. Comme l'écrit Bernard Williams (1990, XV) : "La possibilité de la position ethnographique comme moyen de comprendre une structure étrangère de valeurs qu'on ne partage pas est une donnée de base de la philosophie morale. » 
impose simultanément de juger de la réussite pratique ou de l'échec des initiatives publiques et d'appréhender leurs effets sur les normes collectives et les structures qui les perpétuent. Sans distance critique, la seconde exigence ne peut être atteinte. Le risque existe même d'être trompé par des conceptions qui contribuent à aggraver les problèmes qu'elles prétendent éclairer, par exemple en conférant une signification universelle aux idées qui favorisent la domination d'un groupe ou qui masquent les rapports d'exploitation.

Or, parmi tous les arguments en faveur de la méthode comparative - c'est-à-dire l'étude systématique d'un certain nombre de cas que distinguent l'histoire, la géographie ou quelque autre donnée sociopolitique (Collier, 1993; Ragin, 1987; Lijphart, 1971) -, aucun n'est plus convaincant que la possibilité d'un « décentrement ", d'une lecture nouvelle et critique des expériences et des doctrines propres à une société, par contraste avec d'autres (Campbell, 1988). Quelle que soit l'envergure de l'entreprise de recherche, de la simple étude de cas au vaste programme transculturel, la méthode comparative entend montrer que certaines dynamiques sociales, insaisissables quand on les étudie séparément, surgissent dans une mise en relation généralisée. C'est dire que l'explication comparative, plus que toute autre, permet de faire la part des jugements et des motivations implicites et d'atteindre les faits et les critères, variables d'un cas à l'autre, qui amènent une société à prendre collectivement des responsabilités ou, au contraire, à réduire les problèmes sociaux à des questions individuelles.

Certes, les sociétés ne sont pas des personnes. C'est un abus de langage qui fait dire que les sociétés ont des valeurs ou, pour les mêmes raisons, que les politiques ont des objectifs ou des buts propres (Goodin et Le Grand, 1987, p.4). II est clair qu'en comparant l'action sociale entre des cultures ou des sociétés, nous comparons l'action d'individus et de groupes concrets, agissant pour leur compte et attachant aux résultats attendus un bénéfice qu'ils sont les premiers et parfois les seuls à mesurer. L'avantage de la méthode comparative serait sans doute moindre si elle n'était pas capable de faire ainsi la part des contraintes inhérentes à l'analyse " en contexte », quand il semble que des intérêts précis et des difficultés réelles se profilent derrière les valeurs et les normes. 
À cet égard, il n'est pas inutile de rappeler que les notions de politique sociale et de problème social recoupent ce que la grande majorité des gens identifient en premier lieu à la « politique gouvernementale " - l'éducation, la sécurité du revenu, la santé, le logement, etc. - et qui les touche de plus près que les questions de défense, de politique étrangère ou de politique économique, qui passionnent l'élite (Rose, 1989, p. 5; Hill, 1980, p. 1-8). Le terrain de la politique sociale est celui des interventions directes dans la vie des individus et il est naturel qu'il s'inscrive dans un cadre historique et géopolitique déterminé, méritant une attention minutieuse de la part des chercheurs. Répétons que le but de la comparaison n'est pas d'imposer à l'interprétation une sorte de principe général, en négation des réalités et des milieux, mais de révéler la diversité des codes et des règles qui président à l'élaboration et à la réalisation des politiques sociales, pour mieux comprendre comment les sociétés s'accordent ou se déchirent à leur propos (Forest, 1997b; Meny et Thoenig, 1989, p. 337-341; Anderson, 1979).

Soulignons enfin que la méthode comparative n'est pas exclusivement tournée vers la recherche et la production de connaissances; l'analyse comparée vise aussi l'intervention sociale, en favorisant la transformation des structures et des méthodes d'intervention. Recueillir des données sur les effets concrets de différentes expériences sociales ou économiques, les ordonner et les évaluer, c'est déjà participer à un processus de changement. À l'inverse de la plupart des discussions au sujet de politiques ou de programmes, qui restent souvent abstraites faute d'exemples ou d'applications, l'observation comparative utilise les réalités extérieures comme autant de "laboratoires ». On peut ainsi juger en taille réelle les effets de certaines stratégies de redistribution ou mesurer les conséquences des solutions que les acteurs politiques rêvent d'imposer, dans les différents domaines de la protection et du développement collectifs.

\section{LE PROBLÈME DE L'ÉQUITÉ}

Le problème que pose la réalisation de l'équité dans la vie sociale permet d'éprouver l'approche normative et comparative des politiques sociales, pour des raisons théoriques aussi bien que pratiques. La notion d'équité est en effet indispensable - dans le contexte des théories morales contemporaines - pour saisir d'autres questions importantes telles que la justice, l'égalité ou l'efficience. Elle se trouve également au cœur de la plupart des 
interventions gouvernementales dans le domaine social, car elle constitue une pièce essentielle du système de référence qui légitime l'action des décideurs publics - surtout quand ils doivent s'éloigner d'autres principes d'action dont la fonction de rassemblement s'épuise, au fil des transformations de l'Étatprovidence : fraternité, liberté, prospérité, etc.

Le problème de l'équité est d'abord et surtout un problème d'équilibre entre " ce que chacun est en droit d'attendre et ce qu'on est en droit d'attendre de chacun ", selon la formule de Philippe Van Parijs (1991, p. 241). Dans cette optique, sont équitables toutes les politiques qui donnent accès à des services ou à des avantages en échange d'une " contribution » socialement acceptable, fût-elle aussi symbolique que l'appartenance à la communauté civique ou nationale. II n'est pas nécessaire que les services ou les avantages procèdent du service public, ni que la contribution soit immédiate ou tangible. II suffit que l'accès aux bénéfices de la production sociale soit contrebalancé par un système de prestations obligatoires, auxquelles les personnes sont tenues par le rang, la fortune ou les capacités. En ce sens, l'équité n'est pas un trait distinctif des sociétés politiques développées, mais se rencontre au contraire dans des sociétés très anciennes ou très éloignées des nôtres, quand ce n'est pas dans les unités sociales de base des systèmes modernes familles, groupes de parenté, associations, etc. (Godbout et Caillé, 1995; Lemieux, 1977). C'est d'ailleurs ce trait d'universalité qui nous permet d'ancrer ici la problématique d'une recherche comparative portant sur les valeurs, en dépit de leur sensibilité au contexte historique et social.

L'équité se distingue notamment de l'égalité, qui suppose que l'accès aux services ou les bénéfices qu'on en retire sont les mêmes pour tous, de la justice, qui fait reposer l'accès sur le " mérite » ou la responsabilité, ou même de la moralité publique, qui mène à réprouver certains rapports d'exploitation ou de domination (Le Grand, 1991, p. 107-111). Cependant, il faut noter que la notion de bien commun, qui accompagne la définition de l'intérêt collectif dans la tradition morale héritée de la Grèce et du christianisme, recoupe en fait chacune de ces dimensions (Byrne, 1989, p. 169-175). C'est donc souvent sous cette forme " abrégée » que la société se saisit d'un problème d'éthique sociale. II arrivera qu'une mesure ou une décision qui satisfont le principe d'équité - l'équilibre entre bénéfices et contributions - 
ne respectent pas les critères du bien commun sous d'autres aspects et soient condamnées par l'opinion: certains privilèges fiscaux ou le "patronage », par exemple, qui font facilement l'unanimité contre eux, mais aussi les soins de santé aux fumeurs ou l'indemnisation des responsables d'accidents de la route, comme l'indiquent des débats récents. II arrivera aussi qu'une règle se heurte à la désapprobation générale, même si elle sanctionne une conduite fautive, parce qu'elle atteint les conditions qui semblent nécessaires à la cohésion sociale et morale de la communauté, notamment le sentiment d'une certaine proportionnalité entre ce qui est donné à la collectivité et ce que celle-ci offre en retour.

Certes, dans le monde réel, ni l'équité ni le bien commun ne se réalisent parfaitement; avant d'être un jugement moral, le jugement politique sur ces matières est donc par nécessité un jugement culturel, qui reflète ce que les membres d'une société trouvent tout à la fois raisonnable et convenable de faire (Young, 1994). Quand cet accord est difficile à réaliser et qu'aucune obligation ne s'impose spontanément aux acteurs en présence, comme c'est le cas aujourd'hui pour une grande part des orientations collectives en matière sociale, les rapports de forces se substituent à la négociation et au compromis. Sous le manteau de la justice et de l'intérêt public, l'État lui-même exerce des pressions très fortes en faveur de nouveaux modèles d'organisation, rompant avec les pratiques traditionnelles du secteur public, sans s'inquiéter outre mesure de la façon dont ce virage respecte la sensibilité de la communauté politique.

En fait, l'équité est menacée de quatre façons, qui appellent chacune des réactions différentes de la puissance publique, conformément à l'intuition maintenant classique d'auteurs comme Salisbury (1969) ou Lowi (1972): les politiques déterminent la politique. La menace peut venir d'un problème de redistribution, si la contribution des uns n'est pas à la mesure de leurs moyens ou de leurs talents, ou si le bénéfice des autres n'est pas suffisant pour assurer leur participation à la vie sociale ou même, dans certains cas, à la vie politique ${ }^{3}$.

3. Notons à cet égard qu'un groupe d'experts québécois (Deschênes et collab. 1996, p. 24) a récemment conclu à la nécessité de développer une véritable équité de participation, définie comme: «la chance plus ou moins égale $[. .$.$] de jouer un rôle actif et valorisant dans la vie sociale,$ 
II peut s'agir d'un problème d'allocation, si la distribution des avantages ne se rapporte pas à la contribution exigée, comme on le constate souvent dans les questions de répartition géographique des services publics, mais aussi dans maints assauts contre l'universalité des programmes sociaux. II peut s'agir d'un problème de régulation, si l'accès aux services ou à des avantages est refusé à des personnes qui pourraient y prétendre dans un système régi par d'autres normes, tout aussi acceptables socialement. II peut enfin s'agir d'un problème de mobilisation, si le soutien des bénéficiaires et l'intérêt des bureaucrates qui mettent en œuvre la politique ne parviennent pas à s'équilibrer, de sorte que la demande et l'offre de programmes ne se rencontrent jamais.

La réaction politique traditionnelle à ces difficultés consistait à rétablir l'équilibre des prélèvements et des prestations, en exigeant des contributions plus importantes ou en rationnant les services. Aujourd'hui, on nous dit le plus souvent que la justice distributive est une simple question d'organisation ${ }^{4}$. Or, si le recouvrement plus efficace des impôts et des taxes ou l'élimination systématique de pratiques obsolètes ne peuvent pas nuire, ces mesures ont peu d'effet sur certains des problèmes qui viennent d'être évoqués. La régulation micro-économique de l'utilisation des services, par responsabilisation des usagers, se solde habituellement par un échec, faute de prendre en compte les déterminants culturels et sociaux de la demande de services (De Foucauld, 1995, p. 103; Aaron, 1995). Quant aux réactions des couches moyennes contre le rationnement "négatif » (l'exclusion des mieux nantis) et d'autres formes d'équité verticale, elles ne sont pas toujours de simples manifestations d'égoïsme de classe : elles renvoient à des principes importants du système de valeurs sur lequel est fondée la collaboration dans nos sociétés, particulièrement en matière de fiscalité et de biens

d'en retirer une reconnaissance et de profiter des avantages symboliques et matériels que cette participation peut lui procurer ».

4. Dans un avis récent (Québec, 1995, p. 49), le Conseil de la santé et du bien-être du Québec peut ainsi conclure sans autre précaution que : "Les rapports des commissions d'enquête, les avis d'experts, la documentation scientifique, tout converge: les véritables solutions se trouvent essentiellement du côté de l'amélioration des modes d'organisation du "système de production". " 
sociaux - de chacun selon ses capacités, à chacun selon ses besoins ${ }^{5} \ldots$

Il est inévitable qu'un gouvernement qui s'insinue au centre de la négociation sociale, comme le font assez naturellement les gouvernements modernes, se heurte à des problèmes d'équité, sous l'une ou l'autre des formes qui viennent d'être identifiées. À cet égard, ce qu'on appelle la crise de l'État-providence n'est peut-être pas autre chose que l'émergence (ou la résurgence) de problèmes d'allocation et de régulation dans un système institutionnel où tout a été pensé pour résoudre les questions de redistribution et de mobilisation, depuis les programmes sociaux jusqu'aux partis politiques.

Les questions de politique sociale ne se réduisent donc pas toujours au combat des "riches » et des " pauvres » et à une sorte de jeu à somme nulle entre les intérêts de ces deux groupes. D'une part, les améliorations sociales et l'accès à la consommation ont accru le nombre de ceux qui ne se rangent ni d'un bord ni de l'autre et dont les demandes vont au-delà des mesures de redistribution ou de contrôle social. D'autre part, l'exclusion n'est pas un simple mécanisme économique, mais s'étend à d'autres dimensions de la vie sociale, y compris dans la sphère domestique, où l'expérience de la dépendance et de la violence peut être tout aussi destructrice que celle de la pauvreté. C'est probablement une explication trop simple que rendre compte de ces problèmes par simple référence aux menaces qui pèsent sur l'équité. Mais n'est-il pas légitime de connaître les normes professées et établies dans nos sociétés à l'endroit de conduites ou de situations de ce genre et d'exiger un mode

5. Par équité verticale, on désigne un système d'imposition et d'avantages sociaux dans lequel les contribuables à haut revenu supportent des charges plus lourdes, par l'intermédiaire des impôts ou par celui des règles d'admissibilité aux programmes sociaux (Lefebvre, 1995, p. 17). Sous sa forme extrême, l'équité verticale conduit à réserver les rares dépenses de redistribution aux seuls indigents, en laissant aux autres citoyens l'entière responsabilité de leur bien-être. En fait, même si les interventions gouvernementales n'ont pas toujours servi à corriger les inégalités sociales et que la situation des couches moyennes se compare avantageusement à celle des démunis que l'État tente de protéger, l'uniformité de la couverture sociale est parfois la meilleure garantie que l'ajustement de la capacité contributive et de la couverture sociale ne reflète pas seulement des transferts entre coalitions majoritaires successives (Bélanger, 1987, p. 36). 
d'investigation qui fasse une place à leur discussion publique et systématique?

\section{LES OUTILS D'ANALYSE}

Dans leurs travaux de recherche sur les questions morales, les professionnels de la philosophie politique suivent une méthode argumentative de type axiomatique ${ }^{6}$. Cela consiste à poser quelques principes ou postulats, habituellement confortés par l'intuition ou le sens commun, et à échafauder sur ce substrat une construction théorique plus ou moins ambitieuse, qu'on pourra finalement confronter à la réalité. Par son abstraction et son formalisme, cette démarche est voisine de celle qu'emploient les économistes qui pratiquent la théorie des jeux et, plus largement, de toutes les tentatives qui sont faites pour « remplacer l'étude d'un phénomène concret par celle d'un objet constitué par sa définition » (Attali, 1981, p. 7). Le succès prodigieux d'un John Rawls et de sa Theory of Justice (1971) ne peut être dissocié de cet aspect proprement méthodologique : avant d'être une œuvre de philosophie morale, le livre est d'abord un modèle d'argumentation rationnelle, fondée sur l'examen rigoureux de propositions cohérentes et interdépendantes, réunies dans une même représentation de la justice sociale (Boyer, 1988; Pettit, 1980).

Cela dit, l'application de la méthode axiomatique aux problèmes sociaux ne donne pas toujours des résultats probants, malgré plusieurs tentatives de très bon niveau dans le domaine de la santé (Goold, 1996; Veatch, 1991 et 1981; Daniels, 1985). La nature des questions en examen y est peut-être pour quelque chose, car les « choix tragiques » (Calabresi et Bobbitt, 1978) et autres situations de crise ne s'accommodent pas facilement d'une démarche souvent artificielle, plus occupée de sa propre logique que de l'adéquation du modèle à la réalité. Mais l'obstacle

6. Les spécialistes auxquels nous pensons se réclament presque tous, à quelque degré, de la philosophie analytique, ce qui n'embrasse pas tout le champ de la philosophie politique, mais qui comprend la plus grande partie des travaux d'éthique et de politique. Comme le note Bernard Williams dans la Préface à l'édition française de L'Éthique et les limites de la philosophie (1990, p. 2) : "Ce qui distingue la philosophie analytique du reste de la philosophie contemporaine, [...] c'est une certaine façon de procéder, qui implique l'argumentation, la recherche de distinctions et, dans la mesure où elle s'y emploie et y réussit, un langage relativement simple. » 
majeur réside dans la méthodologie elle-même, qui requiert pour commencer la formulation de principes ou d'axiomes autour desquels un consensus est possible, avant de rechercher les implications de ces définitions et d'apporter une solution aux problèmes posés. Or, il est peu probable que soient jamais réconciliées les positions fondamentales défendues par les différents courants de la philosophie morale - égalité, liberté, justice, compassion, intérêt, utilité, etc. - et sur lesquelles l'accord initial doit être construit. Comme l'a souligné Robert $M$. Veatch, au départ d'un article pourtant très militant en faveur de l'égalité dans l'accès aux soins de santé, " aucune argumentation définitive et rationelle visant à défendre cette interprétation du principe de justice ou n'importe quelle autre n'est vraiment possible (Veatch, 1991, p. 83) ». C'est précisément la difficulté de réunir un consensus collectif autour de valeurs de base qui donne au champ social son caractère politique et qui justifie le recours à l'analyse normative.

En remettant en question les normes morales et la façon dont elles fonctionnent, la méthode axiomatique contribue à mettre en cause bien des prétendues évidences. Mais elle ne peut pas montrer quel but une société doit viser, ni venir à bout des difficultés qui proviennent de la confrontation des valeurs, car la complexité des situations concrètes ne se laisse pas réduire à des postulats sur la justice ou la liberté ${ }^{7}$ (Cassell, 1981). En fait, la convergence des opinions autour de certaines valeurs fondamentales s'apparente moins au consensus rationnel des philosophes ou des scientifiques qu'à un processus de régulation idéologique, notamment quand il s'agit des rapports d'autorité ou de solidarité qui caractérisent la culture politique (Sabatier, 1993; Douglas, 1990). Si ce n'était de cette pression contraignante sur les individus et les groupes, les attitudes et les comportements qu'ils tentent d'élever au rang de normes universelles et d'étendre à tous les aspects de la vie collective se disperseraient sans cesse, à cause de l'expérience historique et de la diversité des intérêts en présence dans nos sociétés.

7. L'Éthique de Nicomaque (Aristote, 1992, p. 63 [II-VII-1]): «Or, il ne faut pas se contenter de cette affirmation générale sur la vertu; il faut aussi que notre théorie soit en harmonie avec les cas particuliers. En effet, en ce qui concerne les actions, qui raisonne en général raisonne dans le vide, tandis que sur les cas particuliers, on a chance d'obtenir plus de vérité. Car les actions ne portent que sur des cas d'espèces; elles doivent donc s'harmoniser avec eux. " 
Pour dire les choses d'une autre manière, il n'existe pas de " contrat social » préalable, d'entente initiale régissant les querelles et les négociations des acteurs sociaux sur des thèmes tels que la protection de l'enfance, l'égalité des conditions de vie ou l'accès aux services de santé. Comme tous les autres traits distinctifs d'une culture, les limites que les acteurs fixent à la responsabilité collective et les obligations qu'ils s'imposent pour la soutenir sont le résultat de leurs relations et de leurs tractations, et ne peuvent être comparées à un " objet » susceptible d'appropriation ou de redistribution (Douglas, 1982; Douglas et Wildavsky, 1982). De telles controverses n'en finissent d'ailleurs jamais, puisqu'on peut toujours les susciter et que les arguments qui les nourrissent puisent au fonds commun de la culture politique. Ce caractère culturel, au sens large, explique aussi pourquoi les questions sociales ne se réduisent pas au problème de la rareté des ressources, du côté de l'économie, ou à celui de leur utilisation, du côté de la gestion : il s'agit bien d'un problème politique, mêlant de manière indissoluble des préférences et des besoins, sans qu'il soit possible de trancher a priori entre les unes et les autres (Sen, 1993, p. 30-54; McLean, 1987, p. 9-10).

Dans l'approche proposée, on fera le postulat que les convictions se traduisent le plus souvent dans des pratiques, tant personnelles que sociales", et qu'elles se répercutent dans la sphère publique. Les normes et les idéaux sont donc sans cesse présents et les décisions politiques reflètent les alliances entre les acteurs qui pensent servir la même cause ou les mêmes intérêts (Bolduc et Lemieux, 1992). Ce dernier point est important: les formes empruntées par le processus politique ou l'allocation des ressources ne coïncident jamais parfaitement avec l'idéologie d'un seul groupe, mais trahissent les changements dans l'état des forces en présence. Le réformateur conservateur qui veut replacer la famille au centre de la vie sociale s'associe tantôt avec ceux qui veulent "rendre le pouvoir à la communauté » et tantôt avec les libéraux qui tentent de limiter l'emprise de l'État sur l'activité sociale. Le projet technocratique trouve ses appuis dans les organismes communautaires, quitte à leur imposer ensuite les critères comptables du secteur marchand. Quant à la façon de retourner l'intervention gouvernementale à l'avantage des acteurs économiques dominants, il ne manque pas 
d'exemples, bien que ces mêmes acteurs protestent à tout venant contre la généralisation de la dépendance et l'inefficacité de l'État (Navarro, 1996).

Ces questions sont difficiles, car elles nous forcent à nous interroger sur la portée réelle de la responsabilité politique, de ses arbitrages et de ses orientations. Les résoudre et le faire de manière à produire tout à la fois l'élargissement de la base de connaissance et la modification des pratiques, telle est cependant la promesse de l'analyse normative, en vertu des conditions posées au départ de ce texte. Se pose aussi le problème de savoir si l'approche choisie est préparée à affronter les difficultés habituelles de l'étude des politiques sociales : la diversité des domaines qu'il faut explorer, l'étendue des besoins, la multiplicité des acteurs, l'intensité des conflits (Landry et Lemieux, 1995). Il est clair qu'on ne peut plus s'en tenir à une description empirique ne cherchant qu'à délimiter des " faits " ou s'en remettre à une théorie générale du social, souvent abstraite au point de n'avoir rien à dire sur le monde réel. Mais il reste à démontrer que l'examen constant et détaillé des valeurs investies dans les politiques publiques peut vraiment favoriser l'intelligence des situations et des ressources sociales, ainsi que l'intégration des connaissances dans la prestation de services ou l'intervention.

\section{UN PROGRAMME DE RECHERCHE}

Dire que deux conditions, le rapport des forces entre des systèmes de valeurs et l'alliance entre des acteurs pour imposer leur vision de la société, sont des éléments déterminants pour comprendre les interventions gouvernementales dans le domaine social revient à dire que les règles éthiques sont aussi des règles politiques - et réciproquement. Une certaine vision des problèmes sociaux les identifie surtout à un défaut d'ajustement, dans une société donnée, entre les mécanismes d'assignation des statuts et la distribution des droits et des richesses (Gil, 1992). Cette vision n'est pas sans intérêt pour comprendre les grands programmes de protection sociale qui sont associés à l'Etat-providence : assurance-maladie, retraites, politiques familiales, sécurité du revenu, etc. Mais l'État ne se limite plus à offrir des services de santé ou à redistribuer des revenus. Sa tutelle se fait aussi sentir dans des domaines qui ne relèvent pas du grand " compromis social » entre travailleurs et patrons, de l'intervention auprès des femmes victimes de violence familiale à la réinsertion des petits délinquants. En fait, une grande partie 
des tâches qui incombent quotidiennement aux éducatrices et aux travailleuses sociales ne se rapportent à un problème d'équilibre entre les statuts et les droits que d'une façon très indirecte et, pour tout dire, assez artificielle. Notre espoir est que la recherche normative aura comme bénéfice principal de mettre en évidence les problèmes de cette nature, à une vaste échelle, et de suggérer des solutions, car elle ne se limite pas à un champ de savoir spécifique (politique, économie, psychologie, etc.), mais vise en quelque sorte la totalité des significations sociales.

En fait, parce que les acteurs associés à l'élaboration ou à la mise en œuvre des politiques sociales se persuadent facilement que les problèmes auxquels ils font face ont une composante morale irréductible (l'intérêt " général »), ils adoptent volontiers une conception assez dirigiste de la recherche sociale, dans laquelle il leur appartient de fixer les critères d'utilité et de développement des connaissances, en fonction de leurs objectifs propres. Toute recherche dans le domaine s'expose ainsi à heurter des intérêts puissants ou à s'immiscer dans des conflits ouverts. Mais le but n'est pas toujours d'accéder au consensus, surtout quand le travail de terrain force la confrontation des idées dominantes avec des faits pratiques qui les contredisent. D'une certaine manière, la tâche de l'analyste consiste plutôt à mettre en évidence le cadre conflictuel qui accompagne toutes les situations de ce genre, autant sur le plan des enjeux que sur le plan des conséquences (Tessier, 1996).

L'approche normative est comparative par nature, nous l'avons souligné dès le départ. II importe donc que les thèmes de recherche retenus présentent un certain degré d'universalité, de manière à faciliter l'examen et la présentation des systèmes de valeurs, dans un premier temps, et la formulation de principes d'action, dans un deuxième temps ${ }^{8}$. II semble par exemple que

8. Dans le cadre du groupe de recherche interaméricain sur les politiques et le travail social, formé de chercheurs du Canada, du Chili et du Mexique, trois thèmes prioritaires ont d'abord été retenus: 1) le rôle des politiques sociales dans le succès et l'échec des stratégies de décentralisation; 2) l'appauvrissement des femmes et le travail; 3) violence et négligence à l'endroit des enfants. II est intéressant de constater qu'en dépit d'écarts évidents entre les trois sociétés, tant sur le plan de la culture que celui du développement économique, il existe une forte convergence dans la perception des problèmes sociaux prioritaires. 
les intervenants sociaux soient un peu démunis, depuis quelques années, quand il s'agit d'évaluer les conséquences «larges » de leurs décisions cliniques - l'agrégation d'une multitude de décisions équitables produit-elle de l'équité au niveau de la société? II est aussi difficile, à l'inverse, d'évaluer les conséquences cliniques des orientations politiques et sociales imposées par l'État, dans tous les champs d'intervention : relations sociales, violence familiale, pauvreté, identité, développement local, etc.

Ce qui apparaît comme une "nécessité » aux yeux des acteurs sociaux, surtout s'ils sont en position dominante, n'engagera pas toujours la communauté dans son ensemble et moins encore les personnes qui rencontrent des difficultés concrètes et particulières. À l'inverse, la reconnaissance de solutions alternatives et de choix différents permettra peut-être de définir de nouvelles règles de priorité, dont l'application peut produire des changements véritables, dans le sens des besoins et des préférences des acteurs concernés.

Pierre-Gerlier FOREST

Professeur agrégé

Département de science politique

Université Laval 


\section{Références bibliographiques}

AARON, Henry J. (1978). Politics and the Professors, Washington (DC), Brookings Institution.

AARON, Henry J. (1995). "Réflexions sur le financement des soins de santé », La Réforme des systèmes de santé - La Volonté de changement, Paris, OCDE, p. 53-65.

ANDERSON, Charles W. (1979). " The Place of Principles in Policy Analysis ", American Political Science Review, vol. 73, $n^{\circ} 3$, p. 711-723.

ARISTOTE (1992). Éthique de Nicomaque, Éditions Jean Voilquin, Paris, GF-Flammarion.

ATTALI, Jacques (1981). Analyse économique de la vie politique, $2{ }^{e}$ éd., Paris, PUF.

BÉLANGER, Gérard (1987). La croissance du secteur public: la recension des écrits économiques, Québec, Commission d'enquête sur les services de santé et les services sociaux.

BOLDUC, Nathalie et Vincent LEMIEUX (1992). Les facteurs influençant la formation des coalitions, Sainte-Foy, Publications du Laboratoire d'études politiques de l'Université Laval, cahier $n^{\circ}$ 92-08.

BOYER, Alain (1988). "La théorie de la justice de John Rawls", dans F. Récanati (dir.), Éthique et Philosophie politique, Paris, Odile-Jacob, p. 21-54.

BURGI-GOLUB, Noëlle (1996). "Égalité, équité : les catégories idéologiques des politiques publiques », Politix - Travaux de science politique, Paris, $n^{\circ} 34$, p. 47-76.

BYRNE, Patrick H. (1989). " Jane Jacobs and the Common Good », dans F. Lawrence (dir.), Ethics in Making a Living: The Jane Jacobs Conference, Atlanta (GA), Scholars Press, p. 169-189.

CALABRESI, Guido et Philip BABBITT (1978). Tragic Choices, New York, Norton \& Co.

CAMPBELL, Donald T. (1988). Methodology and Epistemology for Social Science : Selected Papers, Chicago, University of Chicago Press.

CASSELL, Eric J. (1981). "Do Justice, Love Mercy: The Inappropriateness of the Concept of Justice Applied to Bedside Decisions ", dans Earl E. Shelp (dir.), Justice and Health Care, Boston, D. Reidel, p. 75-82. 
COLLIER, David (1993). "The Comparative Method ", dans A.W. Finitfer (dir.), Political Science: The State of the Discipline II, Washington, DC, American Political Science Association, p. 105-109.

DANIELS, Norman (1985). Just Health Care, Cambridge, Cambridge University Press.

DE FOUCAULD, Jean-Baptiste (1995). Le financement de la protection sociale - Rapport au Premier ministre, Paris, Commissariat général du Plàn.

DESCHÊNES, Jean-Claude et collab. (1996). "Nouvelles orientations des politiques sociales pour une société en mutation; Éléments de diagnostic et jalons de solutions - Réflexions d'un groupe de travail ». Rapport préparé à la demande du ministre de la Santé et des Services sociaux, Gouvernement du Québec.

DOUGLAS, Mary (1982). In the Active Voice, Londres, Routledge \& Paul Kegan.

DOUGLAS, Mary (1990). "Risk as a Forensic Resource », Dædalus, vol. 119, n' 4, p. 1-16.

DOUGLAS, Mary et Aaron B. WILDAVSKY (1982). Risk and Culture, Berkeley, University of California Press.

DUMONT, Fernand (1994). "Approche des problèmes sociaux ", dans F. Dumont, S. Langlois et $\mathrm{Y}$. Martin (dir.), Traité des problèmes sociaux, Québec, Institut québécois de recherche sur la culture, p. 1-22.

FOREST, Pierre-Gerlier (1997a). Six Leçons sur l'analyse normative des politiques sociales, Bordeaux, Cahiers du CERVL.

FOREST, Pierre-Gerlier (1997b). "Les régimes d'équité dans le système de santé du Québec », Analyse de politiques, vol. 23, $n^{\circ} 1$, p. $54-68$.

GIL, David (1992). Unraveling Social Policy, Rochester (VT), Shenkman Publishing.

GodBout, Jacques T. et Alain CAILLÉ (1992). L'Esprit du don, Paris, La Découverte.

GOODIN, Robert E. et Julian LE GRAND (1987). "Introduction », dans R.E. Goodin et J. Le Grand (dir.), Not Only the Poor: The Middle Classes and the Welfare State, Londres, Allen et Unwin, p. 3-16.

GOOLD, Susan D. (1996). «Allocating Health Care: Cost-Utility Analysis, Informed Democratic Decision Making, or the Veil of Ignorance », Journal of Health Politics, Policy and Law, $n^{\circ} 21$, p. $69-98$. 
HILL, Michael (1980). Understanding Social Policy, Oxford, Basil Blackwell et Martin Robinson.

HOGWOOD, Brian W. et Lewis A. GUNN (1984). Policy Analysis for the Real World, Oxford, Oxford University Press.

JENKINS-SMITH, Hank C. (1990). Democratic Politics and Policy Analysis, Pacific Grove (CA), Brooks/Cole.

JENKINS-SMITH, Hank C. et Paul-A. SABATIER (1992). "The Dynamics of Policy-Oriented Learning ", dans P.-A. Sabatier et H.C. Jenkins-Smith (dir.), Policy Change and Learning: An Advocacy Coalition Approach, Boulder, Westview Press, p. 41-56.

LANDRY, Réjean et Vincent LEMIEUX (1995). "Les politiques sociales: dilemmes des interventions de l'État-providence ", dans F. Dumont, S. Langlois et Y. Martin (dir.), Traité des problèmes sociaux, Québec, Institut québécois de recherche sur la culture, p. 997-1011.

LEFEBVRE, P. (1995). "Les politiques familiales au Québec et au Canada. Que faisons-nous? Où allons-nous? », Interface, vol. $16, n^{\circ} 2$, p. $14-27$.

LE GRAND, Julian et Ray ROBINSON (1984). The Economics of Social Problems: The Market versus the State, $2^{\ominus}$ éd., Londres, Macmillan.

LE GRAND, Julian (1991). Equity and Choice: An Essay in Economics and Applied Philosophy, Londres, Harper Collins Academic.

LEMIEUX, Vincent (1977). Le Patronage politique: une étude comparative, Sainte-Foy, Presses de l'Université Laval.

LEMIEUX, Vincent (1980). "Théorie de la communication et analyse des politiques », dans R. Landry (dir.), Introduction à l'analyse des politiques, Sainte-Foy, Presses de l'Université Laval, p. 105-137.

LEMIEUX, Vincent (1989). La Structuration du pouvoir dans les systèmes politiques, Sainte-Foy, Presses de l'Université Laval.

LEMIEUX, Vincent (1995). L'Étude des politiques publiques: Ies Acteurs et leur pouvoir, Sainte-Foy, Presses de l'Université Laval.

LÉVI-STRAUSS, Claude (1983). Le regard éloigné, Paris, Plon.

LIJPHART, Arend (1971). "Comparative Politics and Comparative Method », American Political Science Review, vol. 65, p. 682693.

LOWI, Theodore J. (1972). "Four Systems of Policy, Politics, and Choice », Public Administration Review, vol. 32, n², p. 298310. 
MAJCHRZAK, Ann (1984). Methods for Policy Research, Newbury Park, Sage.

MCLEAN, lain (1987). Public Choice: An Introduction, Oxford, Blackwell.

MENY, Yves et Jean-Claude THOENIG (1989). Politiques publiques, Paris, PUF.

MINOW, Martha (1987). "Justice Engendered 》, Harvard Law Review, 101, p. 11-95.

NAVARRO, Vincenç (1996). "¿Reducir el Estado de bienestar para salvarlo? », El País (Madrid), 23-ix-96, 14.

PETTIT, P. (1980). Judging Justice: An Introduction to Contemporary Political Philosophy, Londres, Routledge \& Kegan Paul.

QUÉBEC. Conseil de la santé et du bien-être (1995). "Un juste prix pour les services de santé », Québec, Editeur officiel.

RAGIN, Charles C. (1987). The Comparative Method: Moving Beyond Qualitative and Quantitative Strategies, Berkeley, University of California Press.

RAWLS, John (1971). A Theory of Justice, Cambridge, Harvard University Press.

ROSE, Richard (1989). Ordinary People in Public Policy: A Behavioural Analysis, Londres, Sage.

SABATIER, Paul-A. (1993). "Policy Change over a Decade or More ", dans H.C. Jenkins-Smith et P.-A. Sabatier (dir.), Policy Change and Learning: An Advocacy Coalition Approach, Boulder, Westview Press, p. 13-39.

SALISBURY, Robert (1969). "An Exchange Theory of Interest Groups », Midwest Journal of Political Science, vol. 13, $n^{\circ} 1$, p. 1-32.

SEN, Armatia (1993). Éthique et Économie, Paris, PUF.

SHENSUL, Jean J. et Gwen STERN (1985). "Collaborative Research and Social Action », American Behavioral Scientist, vol. $29, n^{\circ} 2$, p. $133-138$.

TESSIER, Lise (1996). "Tendencias de las Politicas Sociales en Canadá y el Trabajo Social ». Communication présentée à l'occasion du premier séminaire interaméricain de travail social - Las Politicas Sociales en Chile, México y Canadá. Faculdad de Ciencias Juridicas y Sociales, Universidad de Concepcion, Chili, 8 janvier 1996.

VAN PARIJS, Philippe (1991). Qu'est-ce qu'une société juste? Introduction à la pratique de la philosophie politique, Paris, Seuil. 
VEATCH, Robert M. (1981). A Theory of Medical Ethics, New York, Basic Books.

VEATCH, Robert M. (1991). "Justice and the Right to Health Care: An Egalitarian Account ", dans T.J. Bole III et W.B. Bondeson (dir.), Rights to Health Care, Dordrecht, Kluwer Academic Publishers, p. 83-102.

WASSERSTROM, Richard (1980). Philosophy and Social Issues, Notre Dame, Notre Dame University Press.

WEISS, Carol H. (1986). "The Many Meanings of Research Utilization », dans M. Bulmer (dir.), Social Science and Social Policy, Londres, Allen et Unwin, p. 31-40.

WILLIAMS, Bernard (1990). L'Éthique et les limites de la philosophie, Paris, PUF.

YOUNG, H. Peyton (1994). Equity in Theory and Practice, Princeton, Princeton University Press.

YoUNG, Iris Marion (1990). Justice and the Politics of Difference, Princeton (NJ), Princeton University Press. 